\title{
DESIGN AND CONSTRUCTION OF THE ADVANCED PHOTON SOURCE 352-MHZ RF SYSTEM SWITCHING CONTROL
}

\author{
D. Horan, L. Solita, D. Reigle, N. Dimonte \\ Advanced Photon Source, Argonne National Laboratory
9700 South Cass Avenue, Argonne, Illinois 60439 USA

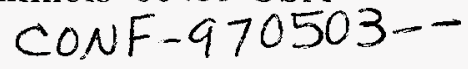 \\ MU 17997

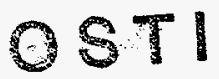

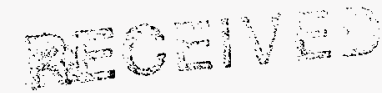

\section{Abstract}

A switching control system has been designed and built to provide the capability of rapidly switching the waveguide and low-level cabling between different klystrons to operate the Advanced Photon Source storage ring in the event of a failure of a klystron system or to perform necessary repairs and preventative maintenance. The twelve possible modes of operation allow for complete redundancy of the booster synchrotron if system and either a maximum of two storage ring if systems to be completely off-line or one system to be used as a power source for an rf test stand. A programmable controller is used to send commands to intermediate control panels which interface to WR2300 waveguide switches and phase shifters, if cavity interlock and low-level if distribution systems, and klystron power supply controls for rapid reconfiguration of the if systems in response to a modeselection command. Mode selection is a local manual operation using a keyswitch arrangement which prevents more than one mode from being selected at a time. The programmable controller also monitors for hardware malfunction and guards against "hot-switching" of the rf systems. The rf switching control system is monitored via the Experimental Physics and Industrial Control System (EPICS) [1] for remote system status check.

\section{RF SYSTEM SWITCHING OVERVIEW}

The Advanced Photon Source has five 1-MW, 352$\mathrm{MHz}$ klystron rf systems installed. The storage ring can operate with either two, three, or four rf stations online, using hybrid combiners/3-dB splitters to operate two rf stations in parallel (see Figure 1). One of the storage ring rf systems can also serve as an operational spare for the booster synchrotron if system, and another can be used as a power source for a 1-MW rf test stand.

The rf stations can be combined in a variety of ways to provide twelve distinct modes of operation that require changes in waveguide $\mathrm{rf}$ circuit configuration, interlock system delegation, and low-level rf signal distribution to permit proper operation of the rf systems. The rf system switching controls execute and verify the required circuit changes for each mode automatically in response to a manual operator command and provide system monitoring functions. Motor-driven WR2300 waveguide switches are used to route the if output of specific rf stations to a designated load, or to take the systems off-line in the event of a failure or for maintenance. Motor-driven WR2300 phase shifters are used to correct for output port phase differences when the 3-dB hybrids are used as combiner/split-

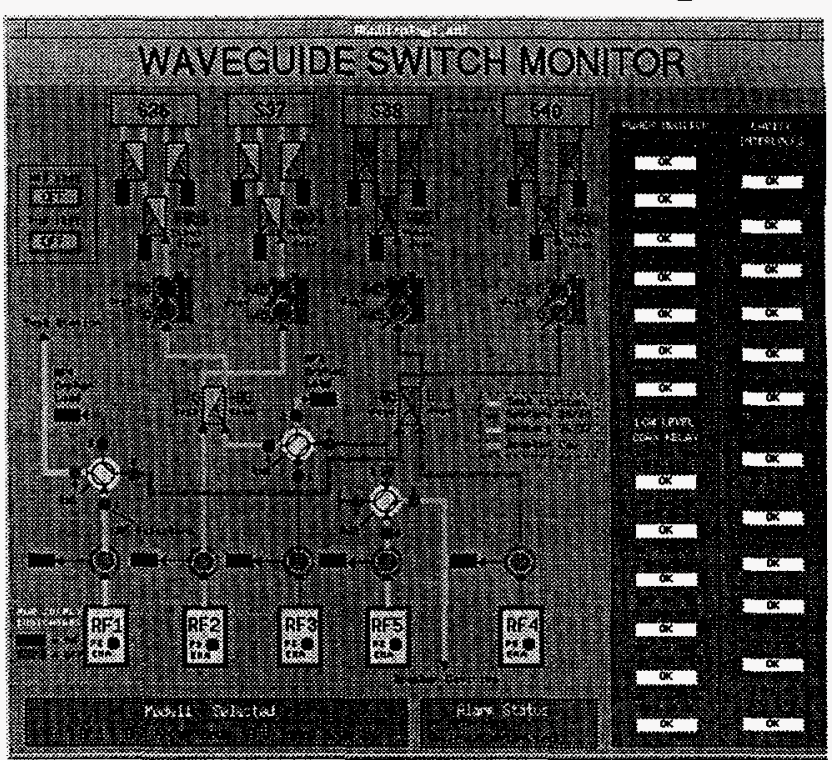

Figure 1: The APS rf system switching control screen.

ters with rf stations in parallel, or as power dividers when a single rf system is used. The cavity and klystron interlock signals for each if system are routed and delegated, respectively, by the switching control system to insure that all interlock circuits required by the accelerator hardware are routed to the rf system supplying the power.

The heart of the if system switching control is an Allen-Bradley programmable logic controller (PLC) that is interfaced to the existing rf system hardware by eight intermediate relay interface panels (see Figures 2 and 3 ). The PLC accepts manual mode requests from operations personnel via a keyswitch panel, executes all necessary system changes required by the selected mode, and monitors overall system status.

\section{SYSTEM CONTROLLER PROGRAM}

The PLC operating program is written to provide a user-friendly interface to the complex task of reconfiguring multiple electrical circuit paths when an rf system operating mode change is requested. It also verifies correct hardware responses to system controller commands and can display error conditions on a local screen as a system troubleshooting diagnostic aid. The program also prevents "hot switching" of if components by verifying that all $\mathrm{rf}$ power is off before any circuits are reconfigured. Mode selection commands are input to the system via the mode

The submitted manuscript has been created by the University of Chicugo as Operator of Argonne National Laboratory (“Argonne") under Contract No. W-31-109-ENG-38 with the U.S. Department of Energy. The U.S. Government retains for itself, and others acting on its behalf, a paid-up, nonexclusive, irrevociable worldwide license in said article to reproduce, prepare derivative works, distribute copies to the public, and perform publicly and display publicly, by or on behalf of the Government. 


\section{DISCLAIMER}

This report was prepared as an account of work sponsored by an agency of the United States Government. Neither the United States Government nor any agency thereof, nor any of their employees, make any warranty, express or implied, or assumes any legal liability or responsibility for the accuracy, completeness, or usefulness of any information, apparatus, product, or process disclosed, or represents that its use would not infringe privately owned rights. Reference herein to any specific commercial product, process, or service by trade name, trademark, manufacturer, or otherwise does not necessarily constitute or imply its endorsement, recommendation, or favoring by the United States Government or any agency thereof. The views and opinions of authors expressed herein do not necessarily state or reflect those of the United States Government or any agency thereof. 


\section{DISCLAMISR}

Portions of this document may be illegible in electronic image products. Images are produced from the best available original docoment 


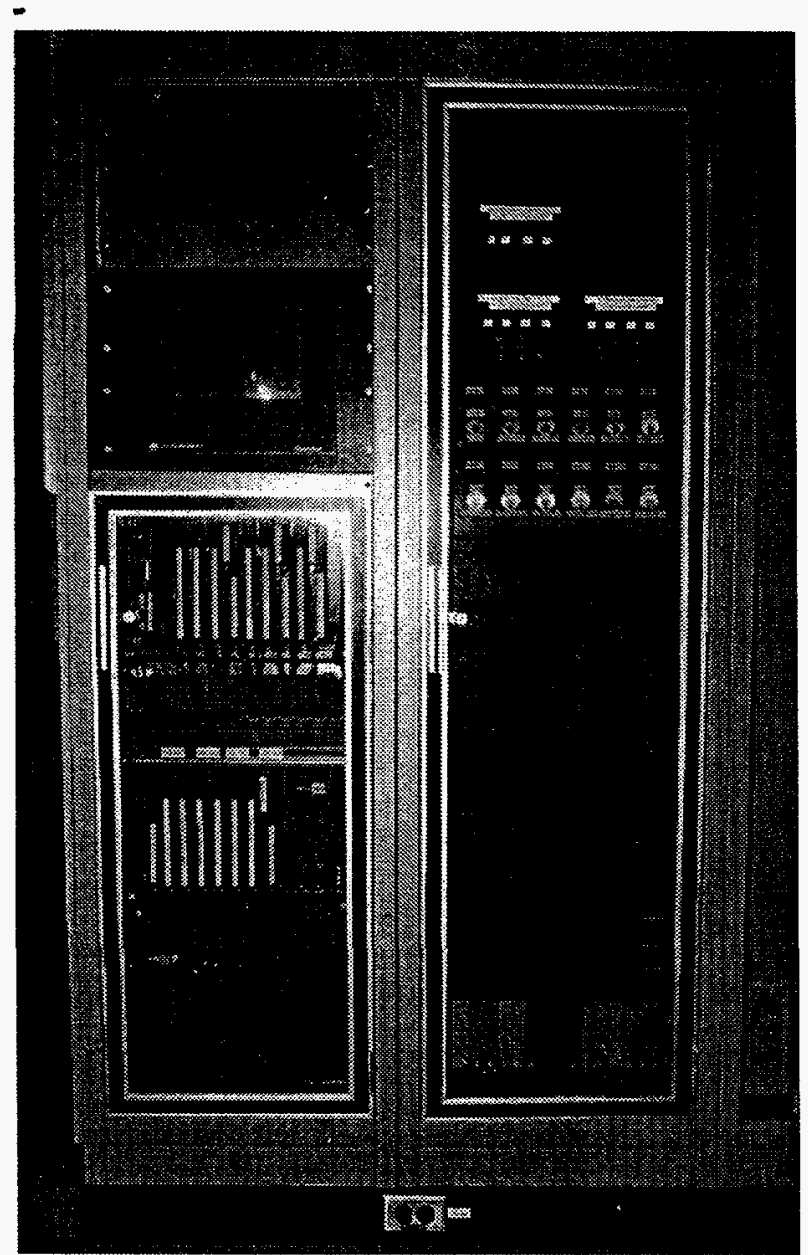

Figure 2: PLC hardware and mode keyswitch.

selection keyswitch panel (see Figure 2), which provides for administrative control of the switching system and prevents selection of more than one mode at a time. The output of the selection switch panel is continuously monitored by the PLC and triggers the PLC to begin the process of mode switching.

The mode selection sequence of events begins with mode-change recognition, where the PLC sends an immediate shutdown command to all rf high-voltage poser supply (HVPS) units, followed by a check for rf presence in all waveguide switch ports to insure all high-power if is off. The PLC then refers to look-up tables to determine the correct configuration of waveguide switches, phase shifters, cavity interlock delegation, rf power monitor delegation, and low-level rf signal distribution required to support the selected mode. The waveguide switches and phase shifters are first commanded to move to their required positions, with a time limit imposed to trigger an error indication and abort the mode change command in the event a mechanism is balky or not moving. Once these movements are complete and home positions match the truth table values, the interlock, low-level of, and power monitor circuits are delegated to match their truth table values for the selected mode. These circuits are configured withn one second, as this action requires merely the activation ol deleyation relays. Once all status indicators match the truth table values for all circuits. the PLC sends an enable command to the of HVPS units that clears them for manual turn-on by system operators.

During rf system operation. the PLC continuously monitors the status of all waveguide switches and phase shitters for unauthorized movement and immediately shuts down the if HVPS units if such a condition is detected. The status of all intermediate relay interface panels is also monitored to detect any unauthorized change in state, which is indicated as an error message on the PLC screen. The rf detector output at the waveguide switch ports is compared with a truth table of expected locations of $\mathrm{rf}$ power for a given mode; any discrepancies generate an error message on the PLC screen.

\section{SOFTWARE AND SYSTEM MONITORING}

The PLC system interfaces rf system input and output devices to a programmable logic processor, which is userprogrammed using an IBM-compatible computer running a "ladder-logic" editor. The editor displays the status of input and output devices as relay contacts and coils. These displays are used to build logical relationships implemented by the program algorithm. Ladder logic is an

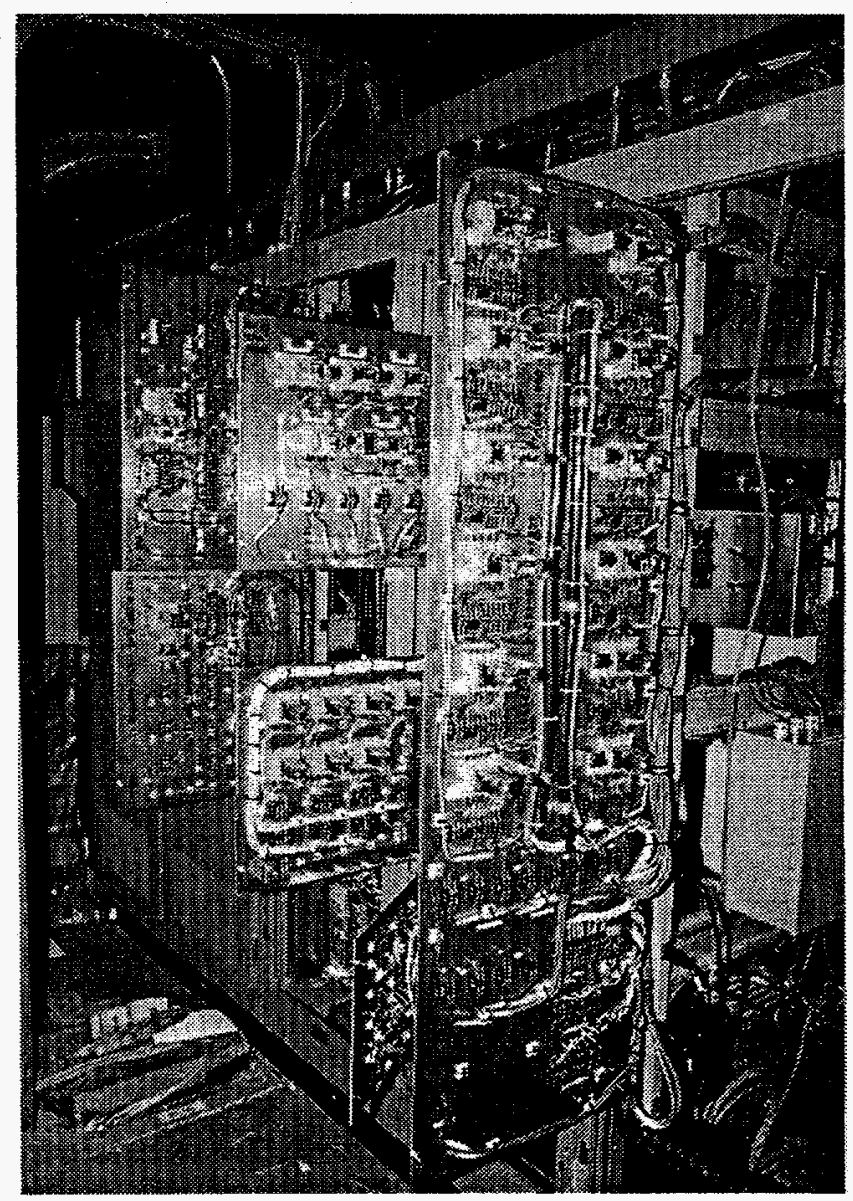

Figure 3: System interface relay panels. 
interpretative programming language and displays PLC states in real time on the computer. Simple logic functions are easily programmed as are more complex functions such as timers, counters, file manipulation, sequencers, and interfaces to display devices like Panelview. The PLC processor constantly calculates a checksum that is unique to each application program.

The Allen-Bradley Panelview provides a local status display of the rf HVPS units, waveguide switches and if detectors, phase shifters, and delegation relays for the cavity interlock, rf power monitor, and low-level coaxial relay systems. Each of these system components is represented on the screen by an identifying icon. As these devices move to their correct position for the operational mode selected, they appear red on the screen. When all devices are in the correct position, the entire status screen is green, and the PLC will enable the five rf HVPS units so they can be energized by operations personnel. The power supplies remain enabled until another mode is selected or the PLC detects that a waveguide switch or phase shifter has moved from its correct position.

For system troubleshooting, diagnostic screens are provided on the Panelview. Faults are latched and timedate stamped to help detect intermittent failures. The operator can select a real-time diagnostic screen for comparison to a reference screen to identify faults. The fault information is retained until another trip is detected. The status of the PLC processor is also displayed, including the program checksum, memory battery status, program scan time, and force status. EPICS is also used to display system status remotely (see Figure 1). This display screen indicates rf system status, waveguide switch and phase shifter position, and present operational mode. The EPICS alarm handler alerts operators to abnormalities in mode status and errors detected in delegation relays for each $\mathrm{if}$ subsystem the PLC controls.

\section{HARDWARE INTERFACE DESIGN}

The PLC communicates with the rf hardware using intermediate relay panels for each subsystem requiring control and monitoring (see Figure 3). These subsystems include waveguide switch control, waveguide phase shifter control, rf HVPS control, cavity interlock contact sum delegation, rf power monitor delegation, low-level if signal routing, if presence detectors, and mode selector keyswitch panel. Each of these panels is supplied 24-volt DC control power from a central distribution chassis that supplies both continuous and switched 24-volt DC outputs. The switched output is enabled only when the PLC watchdog tuner indicates that the operating program is running. The watchdog-switched 24-volt output disables any criti- cal control function of the switching system when the PLC program is not running.

The motorized waveguide switches and phase shifters use 120-volts $\mathrm{AC}$ for motor power; this voltage is accessed locally to each device through individual circuit breakers. This AC power is then looped through local relays which are energized by the PLC only when switch or phase shifter movement is required. In this way, the possibility of unintentional movement of the if hardware through accidental short circuits is reduced. Home-position limit switches inside each waveguide switch and phase shifter provide contact closures which supply the PLC with position indications. The PLC also verifies the correct operation of all other intermediate control relays by monitoring their auxiliary contacts.

The if detector system uses waveguide directional couplers and peak detectors, located close to each waveguide switch port, to generate a proportional DC voltage which is converted to a 4- to 20 -mA current signal for routing to an adjustable alarm module. This module converts the 4- to 20-mA signal to a dry contact closure which supplies an input to the PLC when rf is present in the switch port waveguide. These if detectors are used as a last check for the presence of high-level if in all waveguide switch ports before the PLC sends the command to change switch or phase shifter positions.

\section{CONCLUSION}

The rf switching control system designed and installed at the Advance Photon Source provides accelerator operators with a user-friendly interface capable of reconfiguring complex if systems rapidly in the event of a system failure, thereby reducing accelerator down time. It provides redundancy for both the storage ring and booster synchrotron rf systems and provides remote system status information for diagnostic purposes.

\section{ACKNOWLEDGMENTS}

I would like to recognize Nick Dimonte of the APS Controls Group for his efforts in developing the EPICS display screen for this project, and also Mike Phelan, Gian Trento, and Carl Gonzalez of the APS RF Group for their work building and installing the interface hardware. This work was supported by the U.S. Department of Energy, Office of Basic Energy Sciences, under Contract No. W31-109-ENG-38.

\section{REFERENCES}

[1] L. R. Dalesio, M. R. Kraimer, A. J. Kozubal, "EPICS Architecture," Proc. of ICALEPCS '91, Tsukuba, Japan, KEK Proceedings 92-15, pp. 278-282 (December 1992). 